$11-1-2012$

\title{
Bandemia with normal white blood cell counts associated with infection.
}

\author{
Marci Drees \\ Christiana Care Health System \\ Narrani Kanapathippillai \\ Christiana Care Health System \\ Marc T Zubrow \\ University of Maryland School of Medicine
}

Follow this and additional works at: https://jdc.jefferson.edu/medfp

Part of the Medicine and Health Sciences Commons

Let us know how access to this document benefits you

\section{Recommended Citation}

Drees, Marci; Kanapathippillai, Narrani; and Zubrow, Marc T, "Bandemia with normal white blood cell counts associated with infection." (2012). Department of Medicine Faculty Papers. Paper

83.

https://jdc.jefferson.edu/medfp/83

This Article is brought to you for free and open access by the Jefferson Digital Commons. The Jefferson Digital Commons is a service of Thomas Jefferson University's Center for Teaching and Learning (CTL). The Commons is a showcase for Jefferson books and journals, peer-reviewed scholarly publications, unique historical collections from the University archives, and teaching tools. The Jefferson Digital Commons allows researchers and interested readers anywhere in the world to learn about and keep up to date with Jefferson scholarship. This article has been accepted for inclusion in Department of Medicine Faculty Papers by an authorized administrator of the Jefferson Digital Commons. For more information, please contact: JeffersonDigitalCommons@jefferson.edu. 


\title{
As submitted to:
}

\section{American Journal of Medicine}

\author{
And later published as:
}

\section{Bandemia with Normal White Blood Cell Counts Associated with Infection}

\author{
Volume 125, Issue 11, November 2012, Pages 1124.e9-1124.e15 \\ DOI: 10.1016/j.amjmed.2012.04.039
}

Marci Drees, MD, MS, ${ }^{1,2^{*}}$ Narrani Kanapathippillai, MD, ${ }^{1}$ and Marc T. Zubrow, $\mathrm{MD}^{1,2}$

\author{
${ }^{1}$ Department of Medicine, Christiana Care Health System, Wilmington, DE \\ ${ }^{2}$ Jefferson Medical College, Thomas Jefferson University, Philadelphia, PA \\ * Corresponding author: \\ Marci Drees, MD, MS \\ Department of Medicine \\ Christiana Care Health System
}


4755 Ogletown-Stanton Road, Newark, DE 19718

302-733-5602 (T); 302-733-6386 (F)

mdrees@christianacare.org

Funding source: Christiana Care Health System, Department of Medicine

Conflict of Interest: All authors report no conflicts of interest.

All authors had access to the data and assisted in writing the manuscript.

Article type: Clinical Research Study

Key words: Bands, neutrophilia, infected patients, bacteremia, Clostridium difficile

Running head: Bandemia with normal white blood cell counts 


\section{Abstract}

Background: Measuring immature white blood cell forms ("bands") has been considered clinically unnecessary. We performed this study to determine if elevated band counts, with normal admission total white blood cells, were associated with infection or in-hospital death.

Methods: We performed a retrospective cohort study including all patients admitted to our 2hospital, 1100-bed community-based academic health system in 2009 with normal admission white blood cells $\left(3,800-10,800\right.$ per $\left.\mathrm{mm}^{3}\right)$ who had manual differentials performed. We defined our band groups as normal ( $\leq 10 \%$ bands and other immature cells), moderate (11-19\%), or high ( $\geq 20 \%)$. Via chart review we ascertained vital signs and culture results for all patients with elevated bands and 407 randomly sampled patients with normal bands. Cultures likely to be contaminants were excluded. We used multivariable logistic regression to determine if bandemia was predictive of significant positive cultures or death.

Results: Of 2,342 patients, 167 (7.1\%) had high and 205 (8.6\%) had moderate bands. The mean white blood cell count was 7.5 cells $/ \mathrm{mm}^{3}$, with no difference among groups. Bandemia was associated with increased odds of having any significant positive culture (adjusted OR 2.0, 95\% $\mathrm{Cl}$ 1.3-3.1 for moderate; aOR 2.8, 95\% $\mathrm{Cl}$ 1.7-4.3 for high bands), and of having positive blood cultures (aOR 3.8, 95\% Cl 2.0-7.2 for moderate; aOR 6.2, 95\% Cl 3.2-11.8 for high bands). Patients with moderate or high bands also had increased odds of in-hospital death (aOR 3.2, 95\% Cl 1.7-6.1; aOR 4.7, 95\% Cl 2.4-9.0, respectively).

Conclusions: Even with normal total white blood cells, patients with moderate and high bandemia on admission had significantly increased odds of having positive cultures, including blood cultures, and of in-hospital mortality. 


\section{Introduction}

Infections remain one of the leading causes of death in the United States. ${ }^{1}$ Early recognition of infections permits initiation of multiple therapies, including empiric antibiotics, that have been shown to reduce mortality. ${ }^{2-5}$ While infections often are clinically obvious from history and physical examination, objective evidence is routinely sought, typically including a complete blood count that includes evaluation of total white blood cell number and types using automated methodology. Though sufficient in many cases, the white blood cell count may not yet be increased at the time of clinical presentation. In these circumstances, manual inspection of peripheral blood smears looking for increased band forms (a "left shift") may be beneficial, serving as early indication of serious infections.

In recent decades the band count has fallen out of favor, often considered an unnecessary addition to the total white blood cell and automated differential counts. While band counts may be more useful than total neutrophil counts in neonates, ${ }^{6}$ the usefulness of band counts in patients older than 3 months has been questioned. Bandemia is not specific for infection, but rather is also associated with a variety of inflammatory processes, tissue damage or necrosis, seizures, toxic ingestions, and metabolic abnormalities. In addition, the definition of bands and normal reference ranges can vary between laboratories, and measurement methods are subject to inaccuracy due to sampling bias and subjective identification. ${ }^{7,8}$ Even if accurate, elevated band counts would not be clinically useful to detect early infections, if such infections are already suggested by elevated total white blood cell or automated differentials. While most studies have assessed bandemia as one of several components in a prediction model for infection, ${ }^{9-11}$ one study specifically analyzing patients with normal white blood cell counts found 
that having $19 \%$ or greater bands was poorly sensitive (49\%) for documented positive cultures, but was based on only 33 patients with infections and a similarly sized control group. ${ }^{12}$ Additionally, cost remains an important concern, with one study concluding that obtaining manual differentials routinely was not cost-effective as it affected management in fewer than $3 \%$ of cases. $^{13}$

Because previous studies refuting the role of band counts were underpowered or included patients with both normal and elevated white blood cell counts, we elected to study the specific patient population with normal admission white blood cell counts to determine if, in this specific setting, elevated bands and other immature white blood cells are predictive of microbiologically confirmed infection. Our hypothesis was that in this situation, determining presence of bandemia can be a useful adjunct to total white blood cell count and may allow for earlier suspicion of and empiric treatment for infection. This relatively easily obtained laboratory test could provide valuable information to the clinician, leading to a more rapid initiation of antibiotic therapy and improved patient outcomes.

\section{Methods}

Study design and population. We conducted a retrospective cohort study to identify the association between bandemia and microbiologically-confirmed infection among patients with normal white counts at the time of admission. Christiana Care Health System is a 2-hospital, 1100-bed community-based academic tertiary care health system located in northern Delaware. We selected patients admitted to either hospital in 2009 who had normal white blood cell 
counts $\left(3,800-10,800\right.$ per $\left.\mathrm{mm}^{3}\right)$ and manual differentials performed the time of admission. In our institution, manual differentials are performed on approximately $15 \%$ of all complete blood counts, based on whether the automated differential meets specific criteria (e.g., $>5 \%$ "atypical" lymphocytes or $>15 \%$ monocytes noted), or by clinician request. We excluded patients under 18 years of age and routine obstetrics admissions (i.e., full-term, uncomplicated natural or Caesarian childbirth). In order to avoid including patients with repeated testing that revealed elevated total white blood cells shortly after admission, we also collected follow-up white blood cell counts within the first 24 hours after presentation, and excluded patients with any abnormal white blood cell count within this time frame. Among the remaining patients, we included all with moderate or high band counts (as defined below) and a random sample of 400 patients with normal bands. The size of the normal band group was determined by choosing an approximate 2:1 ratio between the normal group and each of the elevated band groups, to give us $90 \%$ power and alpha of 0.05 to detect a $10 \%$ difference between groups. After the initial selection of the random sample, we determined that a subset of patients lacked any recorded vital signs during the first 24 hours. At this time they were excluded from analysis, and additional patients with normal bands were randomly selected. Ultimately we reviewed 407 patients with normal band counts. The study was approved by the Christiana Care Health System Institutional Review Board.

Data collection. We obtained clinical administrative data, including diagnostic codes, length of stay, and antibiotic utilization, from the electronic medical record for all patients in the cohort. We manually reviewed each chart to collect the first set of vital signs obtained and culture results for cultures obtained within 24 hours of presentation only (from the time first evaluated in the Emergency Department, if applicable). We ascertained the organism, culture source (blood, urine, sputum or wound), and whether multiple cultures with the same organism were 
present, for up to five cultures per patient. We also recorded Clostridium difficile testing results. We did not ascertain possible alternative reasons for bandemia (such as receipt of cytotoxic agents or colony stimulating factors).

Definitions. We calculated band percentage as the percentage of all immature forms (bands, myelocytes, metamyelocytes, and promyelocytes) over the total white blood cell count. We divided the cohort into normal ( $\leq 10 \%)$, moderately elevated (11-19\%), and high ( $\geq 20 \%)$ band groups. The published upper limit of normal at our institution is $3 \%$ bands; however, we used a $10 \%$ upper limit of normal to ensure clinical significance and to match accepted sepsis definitions. ${ }^{14}$ Our primary outcome was a clinically significant positive culture defined as: (1) any urine culture in which at least $10^{5}$ of a single organism were present; (2) sputum culture with moderate or heavy growth of a single organism (excluding those with oropharyngeal contamination); (3) wound or skin cultures with moderate/heavy growth of a single pathogen; (4) C. difficile enzyme immunoassay (EIA) or cytotoxicity assay revealing toxin A and/or B; or (5) blood cultures with growth of a known pathogen (e.g., Staphylococcus aureus, Gram-negative bacilli, Candida species). For coagulase-negative Staphylococci, at least 2 positive cultures obtained via separate blood draws were required. Secondary outcomes included positive blood cultures, C. difficile infection, and in-hospital mortality.

To determine whether patients were ultimately diagnosed with infections, all potential infection-related diagnostic ICD-9 codes were reviewed, and those that lacked specificity (e.g., acute pancreatitis) or relevance to acute inpatient care (e.g., uncomplicated sexually transmitted diseases) were excluded. Subjects whose primary discharge diagnosis code was 
included within the remaining list were considered to have a primary infectious diagnosis. Codes were grouped into "generalized infections" (codes that did not localize to a specific organ system, including bacteremia, viremia, postoperative, transfusion- or central line-related infections and all codes 001.0-139.8 ['Infectious and parasitic diseases', which includes septicemia and all specific bacterial, fungal, viral, and parasitic pathogens]), or by organ system (respiratory, gastrointestinal, skin/soft tissue infection, etc). To determine antimicrobial use within the first 24 and 72 hours, we included all antibacterial and antifungal antibiotics. We did not differentiate antimicrobial agents into different classes.

Microbiology methods. All diagnostic testing was ordered and performed for clinical purposes per patients' admitting clinicians. Blood, sputum, urine and wound cultures were processed using standard CLIA-approved methodology. ${ }^{15}$ C. difficile testing was performed using a twostep process, with EIA for common antigen and toxins A and B, with equivocal results confirmed using cytotoxicity assays.

Statistical analysis. We compared baseline characteristics (demographics, vital signs and initial white blood cell and automated neutrophil counts) for each elevated band group (with the normal band group as comparator) using chi-square and t-tests for categorical and continuous variables, respectively. Wilcoxon rank sum tests were used for variables that were not normally distributed. We performed separate univariate logistic regression analyses to determine whether elevated band counts were predictive of our primary and secondary outcomes (any significant positive culture, positive blood cultures, C. difficile infection, and in-hospital mortality). We performed multivariable logistic regression using other clinically available data at 
time of presentation (age, total white blood cell count, temperature, heart rate, systolic and diastolic blood pressure, respiratory rate) as covariates, with the same separate outcomes as above. We performed all analyses using SAS version 9.2 (SAS Institute, Cary, NC).

\section{Results}

Between Jan 1 and Dec 31, 2009, 2,342 patients met inclusion criteria for the study. Among this population, 167 (7.1\%) patients had high band counts, 205 (8.6\%) had intermediate band counts, and 1970 (84.1\%) had normal band counts. From this latter group, 407 were randomly sampled and included in the study. The three groups had similar initial white blood cell counts (Table 1), but the automated neutrophil count was higher in the moderate and high band groups compared to normal bands. However, all three groups had mean automated neutrophil counts that were above the published upper limit of normal for this test (60\%), and the absolute difference between groups was small.

Compared to those with normal bands, mean heart rate was significantly higher in patients with moderate and high bandemia, and systolic and diastolic blood pressures were significantly lower in the high band group. Temperature and respiratory rate were also higher in the high band group compared to normal bands, but the absolute difference in mean values was small.

As would be expected, patients with moderate or high bands were more likely to have cultures obtained and antibiotics initiated (Table 2). There were 21 patients within the high band group who did not have cultures performed within the first 24 hours. Review of these charts revealed 
that the majority of these patients had trauma, pneumonia, or intraabdominal infections as obvious sources of their bandemia at presentation.

Overall, $214(27.7 \%)$ patients had significant positive cultures and $95(12 \%)$ had positive blood cultures. Positive cultures were significantly more frequent in patients with increased bandemia, with the incidence of any positive culture increasing from $10 \%$ in the normal band group to $32 \%$ in the high band group, while positive blood cultures increased from only $4 \%$ in the normal band group to $26 \%$ in the high band group. Only 13 confirmed cases of $C$. difficile were identified in the cohort, but 7 (54\%) of them were in the high band group. Moderate or high bandemia was also significantly associated with having positive urine cultures, but not with sputum or wound cultures. Interestingly, patients with normal bands were ultimately discharged with an infectious disease-related diagnosis code $30 \%$ of the time, while both elevated band groups had such a code approximately $50 \%$ of the time. In all groups, the majority of these codes fell into the generalized infections category, but the relative proportion varied by group. In the normal band group, generalized infections comprised $59 \%$ of those with a primary infectious diagnosis, with the next most common groups being respiratory infections (11\%) and genitourinary and skin/soft tissue infections (both 10\%). In contrast, among the high band group, generalized infections (including bacteremia and sepsis) was seen in 79\%, and respiratory, genitourinary, and gastrointestinal disease were each seen in only approximately 5\%. Skin/soft tissue infections were only coded $1 \%$ of the time in this group.

Patients with high bands were also more likely to die during their hospitalization, or require discharge to long-term care facilities, compared to patients with normal bands. Among the 88 
patients in the high band group who had primary infectious disease diagnoses, 25 (28\%) died, compared to only 12 (15\%) of 78 patients who lacked a primary infectious diagnosis (OR 2.2, 95\% $\mathrm{Cl} 1.0-4.7)$. Among the normal band group, 10 (53\%) of 19 people who died had a primary infectious diagnosis, and the odds of such a diagnosis among those who died was also significantly elevated (OR 2.6, 95\% $\mathrm{Cl}$ 1.0-6.7). Death was not associated with infectious primary diagnoses in the moderate band group.

In univariate analysis, both moderate and high bands were significantly associated with any positive culture, positive blood cultures, and in-hospital mortality, while only high bands were associated with $C$. difficile infection (Table 3). In multivariable analysis adjusting for vital signs, total white blood cell count and age, increased age was a significant covariate in all analyses, while elevated heart rate was predictive of bacteremia. Having an abnormal temperature on admission $\left(<36^{\circ} \mathrm{C}\right.$ or $\left.>38.3^{\circ} \mathrm{C}\right)$ and decreased systolic and diastolic blood pressures were significantly associated with in-hospital mortality. In terms of specific microbiology, bandemia was most frequently associated with Gram-negative bacilli and Streptococcus pneumoniae bacteremia (Table 4). Polymicrobial infections were also more common in the higher band groups.

\section{Discussion}

Our findings suggest there are selected patients in whom obtaining manual differentials can contribute to the initial diagnostic evaluation. In these patients, elevated band counts could prompt earlier initiation of antibiotics, which has been shown in various patient populations to 
improve mortality. ${ }^{2,4}$ Previous research has challenged the utility of band counts in diagnosing infections, based on low specificity and lack of proven cost-effectiveness. ${ }^{12,13,16-18}$ While many patients with elevated band counts will also have high total white blood cell counts (limiting its additional diagnostic contribution), we specifically limited our analysis to those patients with normal initial white blood cell counts to try to determine if bandemia could provide an earlier suggestion of serious infection. Indeed, in our analyses higher band counts were associated with greater likelihood of having any significant positive culture, but particularly with positive blood cultures and $C$. difficile infection. This association was maintained even when adjusted for other clinical information that is readily available at presentation, such as age and vital signs. Approximately two-thirds of patients in our high band group did have antibiotics initiated within 24 hours, which rose to $75 \%$ within 72 hours, yet they suffered significantly higher all-cause mortality than those patients with moderate/normal bands. However, our analysis was not able or intended to determine whether any delay in appropriate antibiotics contributed to excess mortality.

With regards to particular infections, higher bands were associated with Gram-negative bacteremias, pneumococcal infection and $C$. difficile. $C$. difficile is a well-described cause of significant leukocytosis and bandemia, sometimes prior to the onset of diarrhea, ${ }^{19-21}$ but this study demonstrates that $C$. difficile is also associated with bandemia in the absence of elevated white blood cell counts. This suggests that when encountering a patient with elevated bands but who may lack other objective evidence of infection, empiric antibiotic coverage at a minimum should be directed at Gram-negative organisms and S. pneumoniae. If risk factors or other clinical symptoms suggest antibiotic-associated diarrhea, $C$. difficile should be treated. 
Strengths of this study include that all white blood cell and manual differentials were performed in a single centralized hospital laboratory, which facilitated quality control and standardization needed for consistent, reproducible results. ${ }^{7,22}$ We assessed bandemia in a specific subset of adult patients, those with normal white blood cell counts being admitted to an inpatient service, for whom high band counts might be expected to contribute the most, and our patient groups are otherwise reasonably comparable. As a community-based academic health center, our institution's inpatient population is demographically and socioeconomically diverse, increasing the generalizability of our results. Use of an electronic medical record as well as electronically scanned paper charts enabled us to find admission vital signs and culture results, even when performed prior to hospital admission.

This study also has limitations. Our study population was limited to inpatients, the majority of whom were admitted through the Emergency Department, which may not be generalizable to other patient care scenarios. Manual differentials were not obtained in any standardized fashion, but were based on specific laboratory criteria or clinician preference, which may result in bias toward obtaining band counts for patients who were more likely to have appeared infected. However, we maintained a relatively conservative definition of abnormal immature cells ( $\leq 10 \%$ considered normal), in keeping with accepted sepsis definitions. Secondary analyses that included immature cell percentages of $\leq 6 \%$ considered normal only strengthened our results (data not shown). We did not assess medications (such as cytotoxic agents) or other disease processes (such as myelodysplastic syndromes) that may alter band counts. We relied primarily on culture results, which may represent colonization rather than true infection. 
However, even with this less specific definition we found a significant correlation; excluding cultures more likely to represent colonization (wound and sputum) only strengthened the observed association. We did not determine all of the clinical information available at the time of presentation, such as patient symptoms or radiologic evaluation, and therefore cannot presume that an elevated band count universally provided additional clinical information.

In conclusion, in the setting of normal admission white blood cell counts, the presence of bandemia was associated with increased odds of having significant infection. In particular, Gram-negative or pneumococcal bacteremia and C. difficile infection were common in patients presenting with normal white blood cell counts but elevated bands. While it is likely not costeffective to obtain manual differentials for patients in whom the pretest probability of infection is either very high or very low, this study suggests that determining whether bandemia is present can be a useful adjunct in the initial diagnostic evaluation in selected patients.

Automated methods to accurately determine band counts are lacking, ${ }^{23,24}$ but could potentially improve patient care without significant cost increases. Early empiric antibiotics should be considered even in the setting of normal total white blood cell counts and nonspecific clinical signs and symptoms.

\section{Acknowledgment}

The authors would like to thank James R. Bowen and Claudine Jurkovitz, MD, MPH, Christiana Care Center for Outcomes Research, for their assistance with data extraction for this study. 


\section{References}

1. Kochanek KD, Xu J, Murphy SL, Minino AM, Kung HC. Deaths: Preliminary data for 2009. National vital statistics reports; vol. 59, no. 4. Hyattsville, MD: National Center for Health Statistics. 2011.

2. Kumar A, Roberts D, Wood KE, et al. Duration of hypotension before initiation of effective antimicrobial therapy is the critical determinant of survival in human septic shock. Crit Care Med. 2006;34(6):1589-1596.

3. Nguyen HB, Corbett SW, Steele R, et al. Implementation of a bundle of quality indicators for the early management of severe sepsis and septic shock is associated with decreased mortality. Crit Care Med. 2007;35(4):1105-1112.

4. Rivers E, Nguyen B, Havstad S, et al. Early goal-directed therapy in the treatment of severe sepsis and septic shock. N Engl J Med. 2001;345(19):1368-1377.

5. Houck PM, Bratzler DW, Nsa W MA, Bartlett JG. Timing of antibiotic administration and outcomes for Medicare patients hospitalized with community-acquired pneumonia. Arch Intern Med. 2004;164:637-644.

6. Akenzua GI, Hui YT, Milner R, Zipursky A. Neutrophil and band counts in the diagnosis of neonatal infections. Pediatrics. 1974;54(1):38-42.

7. Cornbleet PJ, RW Novak. Lack of reproducibility of band neutrophil identification despite the use of uniform identification criteria. Lab Hematol. 1995;1:89-96. 
8. Luxmore B, Powell KR, Diaz SR, Novak RW. Absolute band counts in febrile infants: Know your laboratory. Pediatrics. 2002;110(1 Pt 1):e12.

9. Pfitzenmeyer P, Decrey H, Auckenthaler R, Michel JP. Predicting bacteremia in older patients. J Am Geriatr Soc. 1995;43(3):230-235.

10. Bates DW, Cook EF, Goldman L, Lee TH. Predicting bacteremia in hospitalized patients. A prospectively validated model. Ann Intern Med. 1990;113(7):495-500.

11. Mellors JW, Horwitz RI, Harvey MR, Horwitz SM. A simple index to identify occult bacterial infection in adults with acute unexplained fever. Arch Intern Med.1987;147(4):666-671.

12. Ardron MJ, Westengard JC, Dutcher TF. Band neutrophil counts are unnecessary for the diagnosis of infection in patients with normal total leukocyte counts. Am J Clin Pathol. 1994;102(5):646-649.

13. Shapiro M, Hatch RL, Greenfield S. Cost containment and labor intensive tests. the case of the leukocyte differential count. JAMA. 1984;252(2):231-234.

14. Dellinger RP, Levy MM, Carlet JM, et al. Surviving sepsis campaign: International guidelines for management of severe sepsis and septic shock: 2008. Crit Care Med. 2008;36(1):296-327.

15. Garcia LS, ed. Clinical Microbiology Procedures Handbook, 3rd Edition. Washington, D.C.: ASM Press; 2010. 
16. Dutcher TF. Leukocyte differentials. are they worth the effort? Clin Lab Med. 1984;4(1):71-87.

17. Shapiro M, Martin F, Greenfield S. The complete blood count and leukocyte differential count: An approach to the rational application. Ann Intern Med. 1987;106:6574.

18. Connelly DP MM, Crowson TW, Benson ES. The use of the differential leukocyte count for inpatient casefinding. Hum Pathol. 1982;13(4):294-300.

19. Sailhamer EA, Carson K, Chang Y, et al. Fulminant Clostridium difficile colitis: Patterns of care and predictors of mortality. Arch Surg. 2009;144(5):433-9.

20. Wanahita A, Goldsmith EA, Musher DM. Conditions associated with leukocytosis in a tertiary care hospital, with particular attention to the role of infection caused by Clostridium difficile. Clin Infect Dis. 2002;34(12):1585-1592.

21. Wanahita A, Goldsmith EA, Marino BJ, Musher DM. Clostridium difficile infection in patients with unexplained leukocytosis. Am J Med. 2003;115(7):543-546.

22. Cornbleet PJ, Novak RW. Classifying segmented and band neutrophils. CAP Today. 1994;8(5):37-41.

23. Thalhammer-Scherrer R, Knobl P, Korninger L, Schwarzinger I. Automated five-part white blood cell differential counts. efficiency of software-generated white blood cell suspect flags of the hematology analyzers Sysmex SE-9000, Sysmex NE-8000, and coulter STKS. Arch Pathol Lab Med. 1997;121(6):573-577. 
24. Ruzicka K, Veitl M, Thalhammer-Scherrer R, Schwarzinger I. The new hematology analyzer Sysmex XE-2100: Performance evaluation of a novel white blood cell differential technology. Arch Pathol Lab Med. 2001;125(3):391-396. 